\title{
Biological X-ray Fluorescence Microscopy: Advances and Unique Opportunities
}

\author{
S. Vogt ${ }^{1}, *$, S.-C. Gleber ${ }^{1}$, Q. Jin ${ }^{2}$, S. Chen ${ }^{1}$, L. Finney ${ }^{1}$, C. Jacobsen ${ }^{1,2}$, M. Ralle ${ }^{3}$, C. Fahrni ${ }^{4}$, and D. \\ Mustafi $^{5}$ \\ ${ }^{1}$ X-ray Science Division, Argonne National Laboratory, Argonne, USA \\ 2. Department of Physics and Astronomy, Northwestern University, Evanston, USA \\ ${ }^{3 .}$ Department of Molecular and Medical Genetics, Oregon Health \& Science University, Oregon, USA \\ ${ }^{4}$ School of Chemistry and Biochemistry, Georgia Institute of Technology, Atlanta, USA \\ ${ }^{5}$ Department of Radiology, University of Chicago, Chicago, USA \\ *email:svogt@aps.anl.gov
}

Trace elements, in particular metals, play a significant role in most known life forms. It is estimated that one-third of all known proteins contain metal cofactors, and the majority of these function as essential metalloenzymes catalyzing biochemical reactions. Trace metals are increasingly recognized as having a critical impact on human health both in their natural occurrence and via therapeutic drugs, and in diseases such as Alzheimer's or Wilson's disease. Quantitative study of the distribution of trace elements on the cellular and subcellular level provide important information about functions and pathways of metalloproteins and therapeutic approaches, especially in conjunction with the local chemical state of the elements of interest.

Hard x-ray fluorescence microscopy is a powerful technique to map and quantify element distributions in biological specimens such as tissues, cells and bacteria. It provides attogram sensitivity for transition metals like $\mathrm{Cu}, \mathrm{Zn}$, and other biologically relevant trace elements, combined with the capability to penetrate whole cells and thick tissue sections. Elemental content is measured directly by using the characteristic fluorescence of atoms excited by the microfocused X-ray beam, without the need for element-sensitive dyes. Typically, 10-15 elements are mapped simultaneously, leading to precise elemental colocalization maps. In addition, the possibility of selecting the incident X-ray energy enables microspectroscopy and chemical state mapping to determine the speciation of elements of interest. Phase contrast allows one to sensitively detect specimen structure, and combine structural information of the sample with trace elemental content.

Lateral spatial resolutions of $200 \mathrm{~nm}$ are routinely achieved, $30 \mathrm{~nm}$ has been demonstrated. In addition, the large penetration depth of hard x-rays permit the analysis of 'thick' samples, and by acquiring numerous 2D projections at different specimen angles, 3D elemental distributions can be reconstructed and visualized. Advances in instrumentation, such as faster detectors, better optics, and improved data acquisition strategies are fundamentally changing the way experiments can be carried out, giving us the ability to more completely interrogate samples, at higher spatial resolution, with larger field of view, higher throughput and better sensitivity. For example, Fig. 1 shows plunge frozen and freeze dried HeLa cells scanned at 0.2 micron spatial resolution across a $\sim 300$ micron wide field of view.

We will report on the capabilities of existing microprobes, in the context of biological specimens, and discuss methods we have implemented, including data analysis, phase contrast techniques to visualize soft tissue, fast fly-scanning, and X-ray fluorescence micro-tomography. We will demonstrate their application in several ongoing studies, ranging from the visualization of trace elemental content in a mouse model of Wilson's disease, to the investigation of novel contrast agents for imaging. We will also 
discuss challenges and opportunities for future scientific applications and instrumentation, in particular with regards to ever faster detectors, better optics, and improved data acquisition strategies.

[1] Use of the Advanced Photon Source, an Office of Science User Facility operated for the U.S.

Department of Energy (DOE) Office of Science by Argonne National Laboratory, was supported by the U.S. DOE under Contract No. DE-AC02-06CH11357. This work was also supported by a grant from the National Institutes of Health-R01 GM104530.
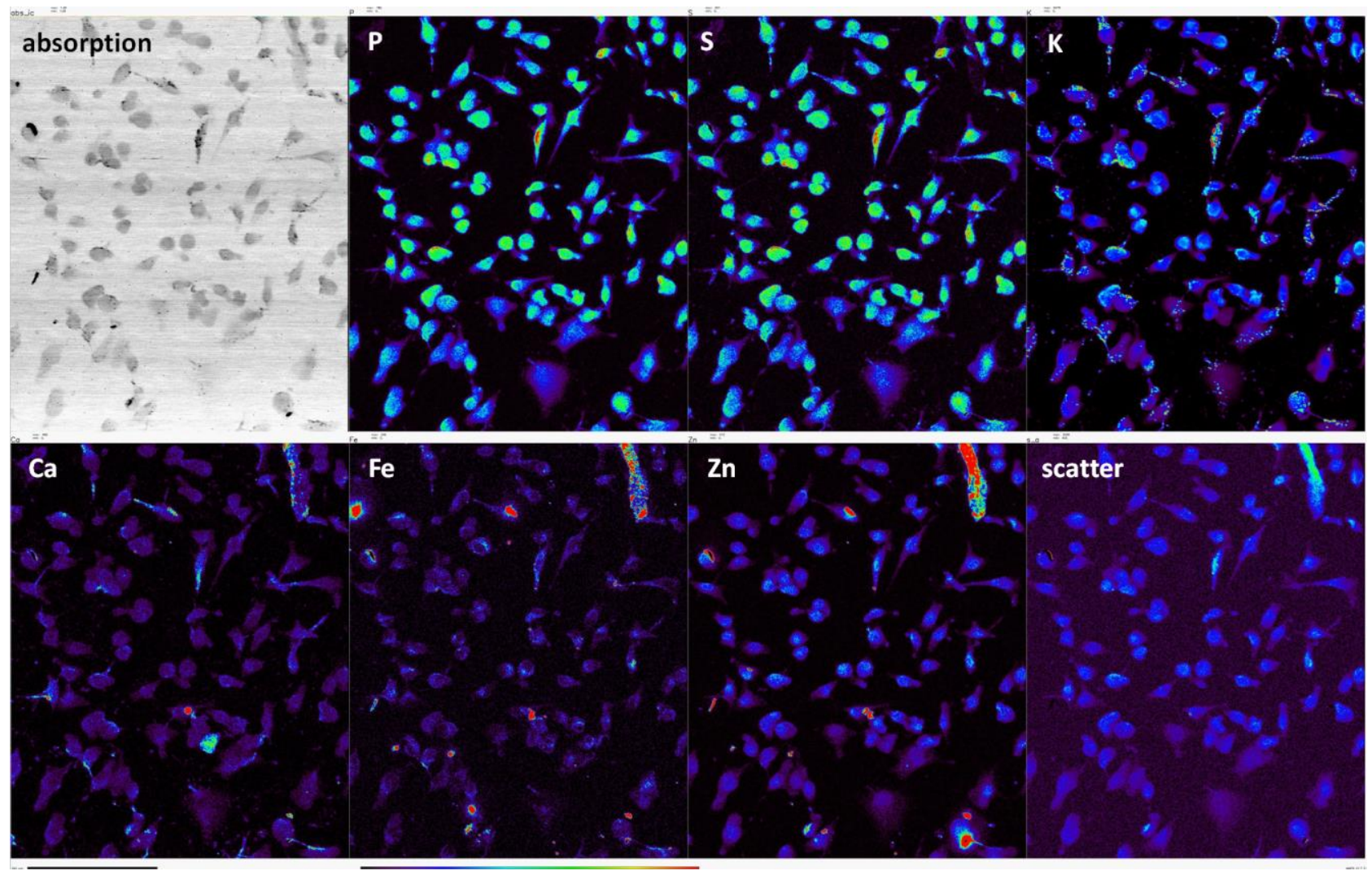

Figure 1. Absorption contrast image as well as elemental maps of $\mathrm{P}, \mathrm{S}, \mathrm{K}, \mathrm{Ca}, \mathrm{Fe}, \mathrm{Zn}$ and a map of the overall scattering signal from a 260x320 micron wide sample of HeLa cells. 